\title{
ANALYSIS OF MULTIPLE BIRTH RATES IN JAPAN \\ VII. RATES OF SPONTANEOUS AND INDUCED TERMINATIONS OF PREGNANCY IN TWINS
}

\author{
Yoko ImAIzUmi, ${ }^{1}$ Akio AsaKa, ${ }^{2, *}$ and Eiji InOUYE ${ }^{3}$ \\ ${ }^{1}$ Institute of Population Probiems, Ministry of Health and Welfare, \\ Tokyo 100, Japan \\ ${ }^{2}$ Institute of Brain Research, University of Tokyo School of Medicine, \\ Tokyo 113, Japan \\ ${ }^{3}$ Institute for Developmental Research, Aichi Prefectural Colony, \\ Aichi 480, Japan
}

\begin{abstract}
Summary The effect of maternal age, birth order, gestational age and total birth weight in twins on stillbirth rate of twins was separately analysed for spontaneous and induced termination of pregnancy. The stillbirth rates in twins and in the general population indicated a U-shape pattern with respect to maternal age for spontaneous and induced terminations of pregnancy. As to the rate of induced termination of pregnancy twins and the general population indicated no marked difference in each maternal age groups.

The rate of spontaneous termination of pregnancy in twins increased with gestational age up to 5 completed months and decreased as the gestational age increased, whereas the rate of induced termination of pregnancy decreased as the gestational age increased.

The highest overall stillbirth rate in twins was seen in the lowest total birth weight and the rate decreased as the total birth weight was increased.

The effects of gestational age, maternal age and birth order on the rate of spontaneous termination of pregnancy were relatively small and the effect of total birth weight of twins was the largest.
\end{abstract}

\section{INTRODUCTION}

In the first report of the present series Imaizumi and Inouye (1979) reported that stillbirth rates of monozygotic (MZ) and dizygotic (DZ) twins were higher in young and old maternal age groups in Japan. Imaizumi et al. (1980a) subsequently analysed such factors affecting stillbirth rate of twins as secular change, zygosity,

Received January 13, 1982

* Present address: Department of Mental Health, University of Tokyo School of Medicine, Tokyo, Japan. 
sex, birth order at twin delivery, birth order of the twin pair and gestational age. Asaka et al. (1980) presented data on the effect of birth weight, and Imaizumi et al. (1980b) analysed the effect of month of birth. The latter authors also studied stillbirth rates separately for spontaneous and induced termination of pregnancy and analysed the rate differences among various occupations of the heads of the household.

The present study further analyses the effect of maternal age, birth order, gestational age and total birth weight of twins on stillbirth rate of twins separately for spontaneous and induced termination of pregnancy. It also examines the relative contribution of these factors to the stillbirth rate of twins.

\section{MATERIALS AND METHODS}

Source of data is "Survey on Socio-economic Aspects of Vital Events-Plural Births in 1975" (Health and Welfare Statistics and Information Department, Ministry of Health and Welfare). This consists of two surveys, A and B, and the present study utilizes only Survey A, which provides data on 12,392 twin pairs taken from certificate records on the live birth and fetal death (after the beginning of the fourth month of fetal life) for all of Japan in 1974. Details of the survey have been reported elsewhere (Imaizumi and Inouye, 1979; Imaizumi et al., 1980a). Among items included in Survey A following ones were used in the present analysis: maternal age, gestational age, the numbers of previous pregnancies and live births, sex of twins, live birth or fetal death, spontaneous or induced termination of pregnancy in case of fetal death, and weight at birth.

In the following analysis, the stillbirth rate was taken among twin individuals.

\section{RESULTS}

Stillbirth rates of spontaneous and induced termination of pregnancy in twins

Table 1 shows the number and the rates of twin individuals ended in spontaneous or induced termination of pregnancy in 1974 according to maternal age. Also shown in Table 1 are corresponding rates in the general population in the same year, and the rates are summarized in Fig. 1. The rates in twins and in the general population indicated a U-shape pattern with respect to maternal age. Proportion of overall spontaneous termination of pregnancy among total fetal deaths was $87 \%$ for twins and $68 \%$ for the general population, and the rate of overall spontaneous termination of pregnancy in twins $(0.115)$ was 3.3 times as high as that in the general population (0.035). If examined for each maternal age group the rate in twins is 3.8 times as high as that in the general population in the group of 25-29 years, which lies at the bottom of the U-shape, while the former is only 1.1 times as high as the latter in the group of 40 years or more. As to the rate of induced termination of pregnancy twins and the general population indicated no marked difference in each maternal age group. 

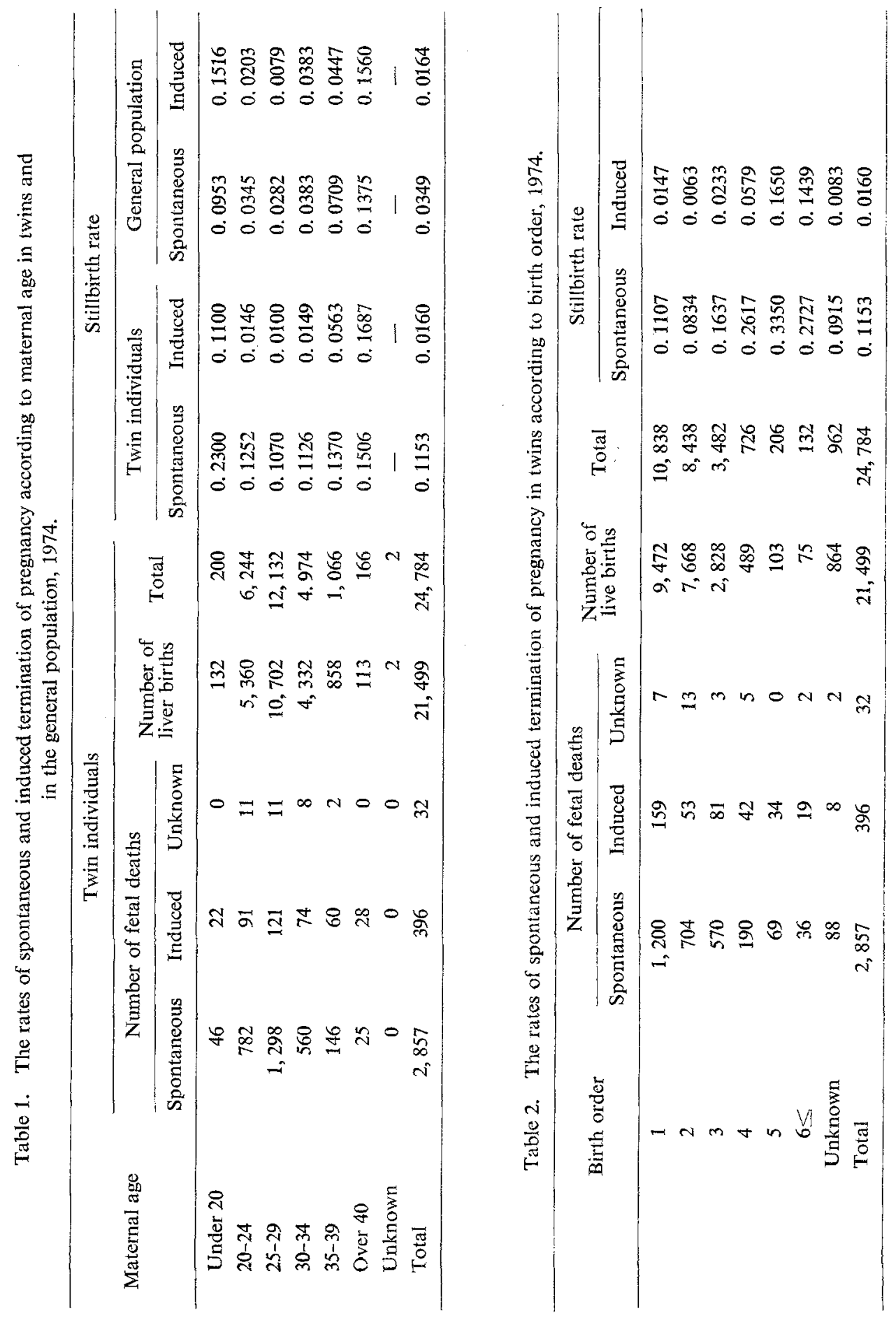


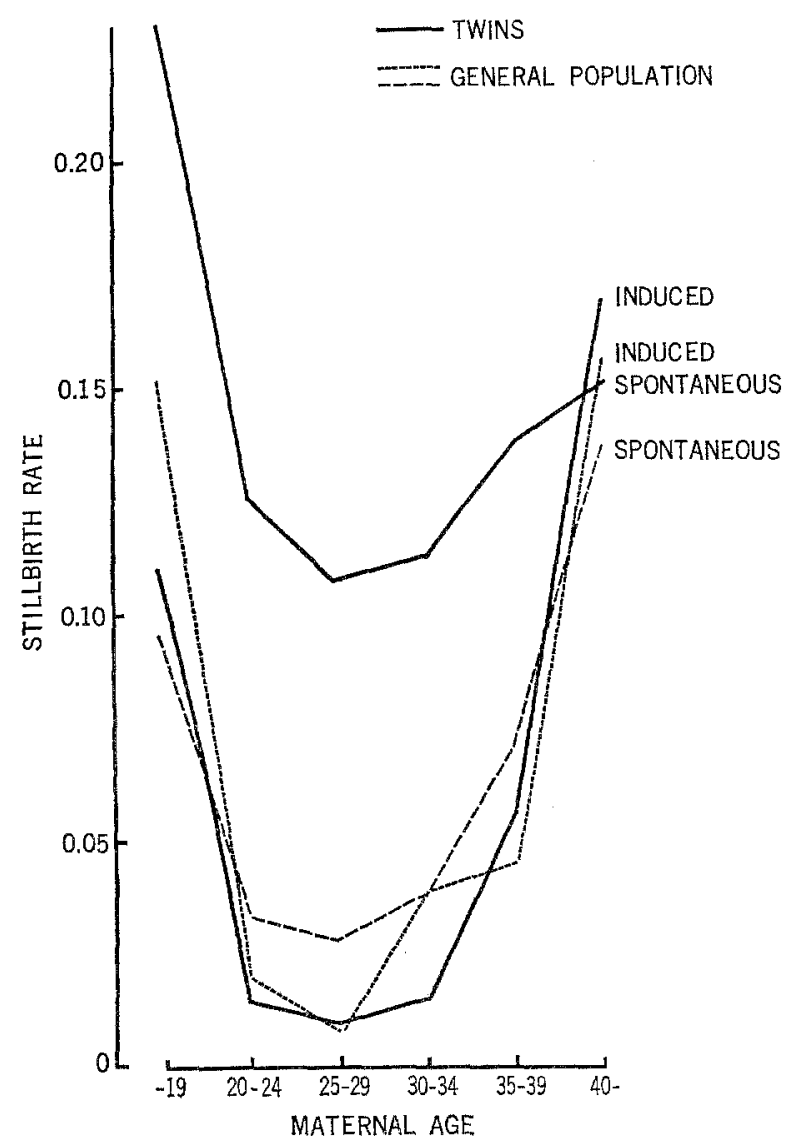

Fig. 1. Stillbirth rates of twins and the general population for spontaneous and induced terminations of pregnancy by maternal age in 1974 .

Table 2 shows the number and the rate of twin individuals ended in spontaneous or induced termination of pregnancy according to birth order. The birth order is defined as the numerical order of all children and fetuses (live-born and prenatally died after five completed months of gestation), where a delivery of twins was counted once. For both types of fetal deaths the rates decreased from the first to the second birth, then increased up to the 5 th order but again decreased in the next higher birth order.

Table 3 shows the rates of spontaneous and induced termination of pregnancy in twin individuals according to gestational age. The rate of spontaneous termination of pregnancy increased with gestational age up to 5 completed months and decreased as the gestational age increased, whereas the rate of induced termination of pregnancy decreased as the gestational age increased. 


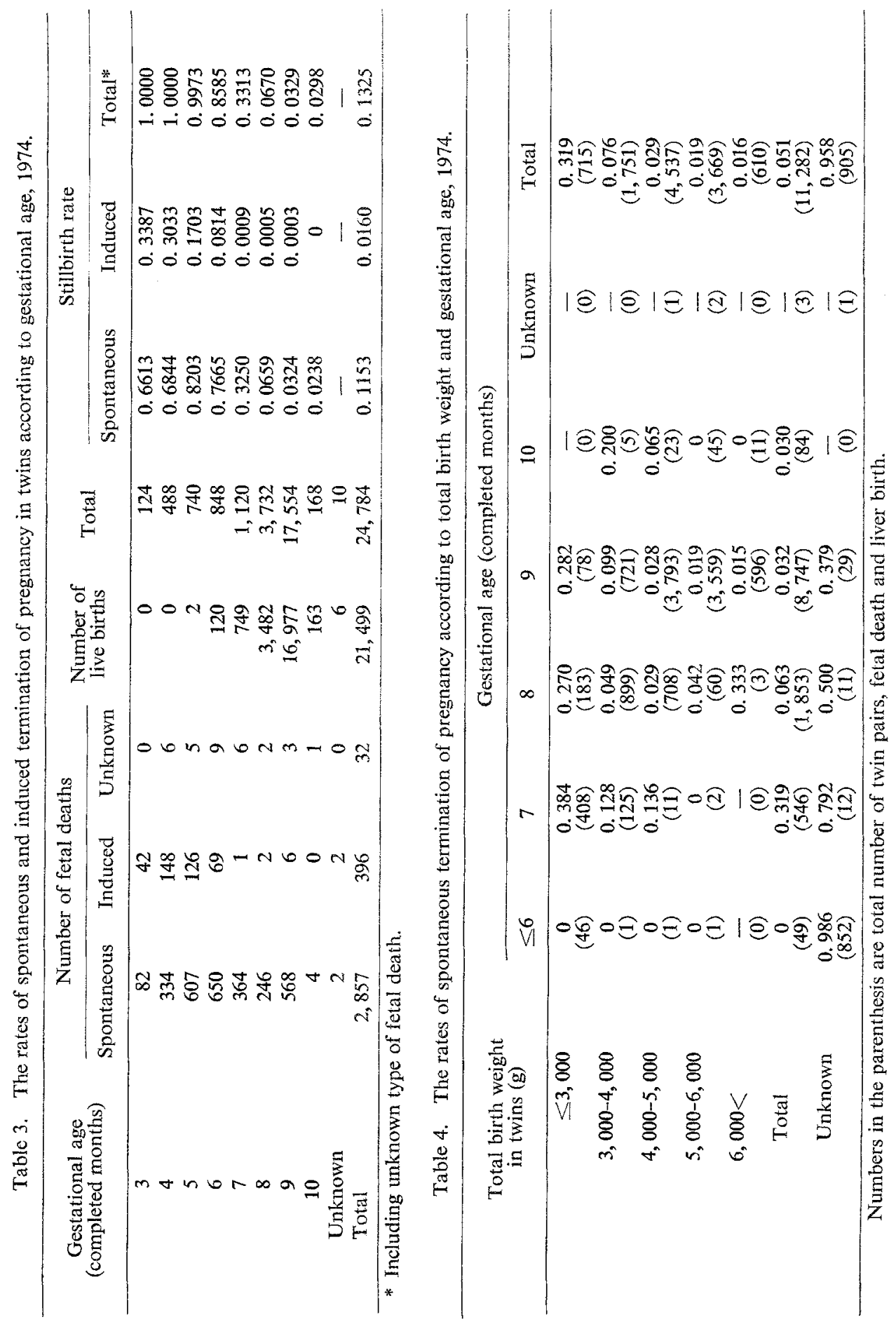

Vol. 27, No. 3, 1982 
Stilibirth rate of twins and total birth weight

Table 4 shows the rate of spontaneous termination of pregnancy according to the total birth weight of the twins and gestational age. The highest overall rate $(0.319)$ was seen in the lowest total birth weight (less than or equal to $3,000 \mathrm{~g}$ ) and the rate decreased as the total birth weight was increased. The lowest rate was 0.016 for the highest total birth weight (more than 6,000 g). Excluding gestational age group of six months or less, where birth weight is unknown in the majority of twin pairs, and the classes with the number of subjects less than ten, the decrease of the rate with gestational age (Table 3 ) is seen in most birth weight groups. For each gestational age group the rate tends to decrease as birth weight increase, as expected.

Table 5 shows the rate of spontaneous termination of pregnancy according to the total birth weight of twins and maternal age. Again excluding classes with the number of subjects less than ten, no single birth weight group indicated clear Ushape pattern with respect to maternal age (see Table 1, Fig. 1). Rather, in the group of the lowest total birth weight the rate decreased as the maternal age was increased. A tendency is seen that in most maternal age groups the rate decreases as birth weight increases, as expected.

Table 6 shows the rate of spontaneous termination of pregnancy according to the total birth weight of twins and birth order. Excluding classes with the number of subjects less than ten increase of the rate from second birth order (Table 2) was seen in most birth weight groups. For each birth order group the rate decreased as birth weight increased, as expected.

\section{DISCUSSION}

As seen in Table 1 it was indicated that there was no difference of the rates of induced termination of pregnancy between twins and the general population, while the rate of spontaneous termination in twin pregnancy was more than three times as high as that in the general population. Further analysis indicated the rate of spontaneous termination in twin pregnancy was dependent on all four factors analysed: total birth weight of twins, gestational age, maternal age and birth order. Then, multiple discriminant analysis was performed to see the relative contribution of total birth weight of twins $\left(\mathrm{X}_{1}\right)$, gestational age $\left(\mathrm{X}_{2}\right)$, maternal age $\left(\mathrm{X}_{3}\right)$ and birth order $\left(\mathrm{X}_{4}\right)$ on the rate of spontaneous termination of pregnancy in twins. Discriminant function obtained was

$$
\mathrm{Y}=-0.8075 \mathrm{X}_{1}-0.2297 \mathrm{X}_{2}-0.1583 \mathrm{X}_{3}+0.3090 \mathrm{X}_{4}
$$

where $Y$ is discriminant function, and constant values are the standardized discriminant function coefficients for variables of $X_{1}$ to $X_{4}$, respectively. Negative effects are seen for total birth weight, gestational age and maternal age, whereas a positive 

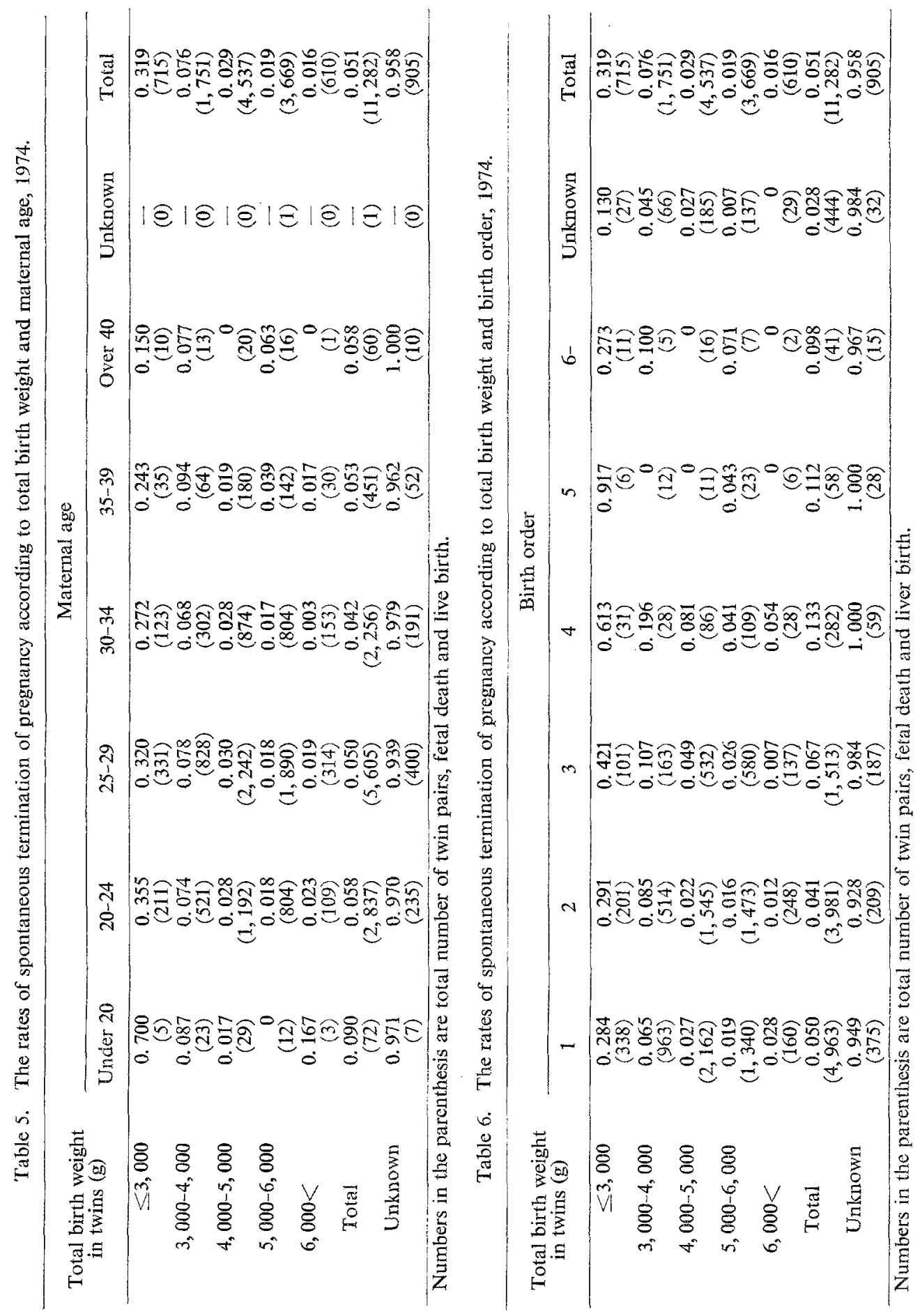
effect is seen for birth order. The effects of gestational age and maternal age were relatively small and the effect of total birth weight of twins was the largest.

An interesting finding was that the rates of both spontaneous and induced terminations in twin pregnancy run parallel according to maternal age as well as birth order (Table 1, Fig. 1, Table 2). This suggests the presence of a common cause either resulting in spontaneous termination or necessitating induced termination in twin pregnancy, despite a popular notion that in Japan induced termination of pregnancy is performed widely on socio-economic reasons.

\section{REFERENCES}

Asaka, A., Imaizumi, Y., and Inouye, E. 1980. Analysis of multiple births in Japan. I. Weight at birth among 12,392 pairs of twins. Jpn. J. Human Genet. 25: 65-71.

Imaizumi, Y., and Inouye, E. 1979. Analysis of multiple birth rates in Japan, I. Secular trend, maternal age effect, and geographical variation in twinning rates. Acta Genet. Med. Gemellol. 28: 107-124.

Imaizumi, Y., Asaka, A., and Inouye, E. 1980a. Analysis of multiple birth rates in Japan. II. Secular trend, and effect of birth order, maternal age and gestational age in stillbirth rate of twins. Acta Genet. Med. Gemellol. 29: 223-231.

Imaizumi, Y., Asaka, A., and Inouye, E. 1980b. Analysis of multiple birth rates in Japan. V. Seasonal and social class variations in twin births. Jpn. J. Human Genet. 25: 299-307.

Japan, Ministry of Health and Welfare. 1976. Vital Statistics, 1974. Health and Welfare Statistics and Information Department, Ministry of Health and Welfare, Tokyo.

Japan, Ministry of Health and Welfare. 1977. Survey on Socio-economic Aspects of Vital EventsPlural Births, 1975. Health and Welfare Statistics and Information Department, Ministry of Health and Welfare, Tokyo. 